\title{
Intermédialités
}

Histoire et théorie des arts, des lettres et des techniques

Intermediality

History and Theory of the Arts, Literature and Technologies

\section{L'abdication devant l'image? Figures du manque, de la disparition et du deuil dans les oeuvres récentes de Sophie Calle}

\section{Cécile Camart}

Numéro 7, printemps 2006

Filer (Sophie Calle)

Shadowing (Sophie Calle)

URI : https://id.erudit.org/iderudit/1005516ar

DOI : https://doi.org/10.7202/1005516ar

Aller au sommaire du numéro

Éditeur(s)

Centre de recherche sur l'intermédialité

ISSN

1705-8546 (imprimé)

1920-3136 (numérique)

Découvrir la revue

Citer cet article

Camart, C. (2006). L'abdication devant l'image? Figures du manque, de la disparition et du deuil dans les oeuvres récentes de Sophie Calle.

Intermédialités / Intermediality, (7), 49-66. https://doi.org/10.7202/1005516ar
Résumé de l'article

Lors de son exposition rétrospective au Centre Pompidou en 2003, Sophie Calle a dévoilé des oeuvres inédites, créées pour l'occasion. Cette étude, qui se nourrit aussi d'une collaboration curatoriale et éditoriale avec l'artiste depuis 2002 , considère les problématiques originales abordées par les pièces Unfinished (1988-2003), Une jeune femme disparaît (2003), Voyage en Californie (2003) et Douleur exquise (1984-2003). L'oeuvre n'est plus envisagée d'un simple point de vue intertextuel et narratif. Le postulat est celui d'une autonomie renouvelée de l'image, qui révèle l'omniprésence des métaphores de la disparition, du manque et de la perte. La relique pourrait devenir ici l'expression d'un impossible deuil, en filigrane, dans l'oeuvre. 


\title{
L'abdication devant l'image?
}

\section{Figures du manque, de la disparition et du deuil dans les œuvres récentes de Sophie Calle}

\author{
Cécile Camart
}

E

n 2003, le Centre Pompidou consacrait à Sophie Calle une importante la critique d'art. L'itinérance internationale des œuvres donna l'occasion à l'artiste de mettre à l'épreuve ses récentes créations afin de leur allouer une forme plus stable1. La proportion notable des pièces inédites spécialement conçues pour l'événement, telles Unfinished (2003), Une jeune femme disparaît (2003), Voyage en Californie (2003) et Douleur exquise (1984-2003), forme une matière qui demande à être considérée ${ }^{2}$. Une nouvelle phase semble de fait s'annoncer dans son travail, avec l'apparition de pièces plus réflexives focalisées sur la méthode et sur les modèles propres de l'artiste. En effet, le sens profond des productions de Sophie Calle déborde les apparences trompeuses d'un seul badinage intertextuel avec les frontières de la fiction et de la réalité. Tandis que l'œuvre est largement diffusée sous une forme éditoriale, une lecture strictement textuelle et narrative omettrait d'appréhender les pièces dans leur intégrité, puisque l'artiste les conçoit

1. L'exposition Sophie Calle. M'as-tu vue s'est déroulée du 19 novembre 2003 au 15 mars 2004 au Centre Pompidou, Paris. Elle a voyagé avec quelques varia à l'Irish Museum of Modern Art de Dublin (du 23 juin au 15 août 2004), au Martin-Gropius-Bau de Berlin (du 10 septembre au 13 décembre 2004) et au Ludwig Forum für Internationale Kunst de Aachen (du 29 janvier au 24 avril 2005).

2. Cette étude s'est nourrie de mon «observation participative» entre 2002 et 2005 , en tant que coordinatrice éditoriale du catalogue de l'exposition Sophie Calle. M'as-tu vue (Christine Macel (éd.), Paris, Éditions du Centre Pompidou, Éditions Xavier Barral, 2003) et commissaire de son exposition au Ludwig Forum für Internationale Kunst (Aachen, Allemagne, 2005). 
pour le musée et songe à leur mise en scène dans l'espace³, bien plus qu'à la mise en page dans un livre. C'est pourquoi la description attentive des dispositifs d'images et des installations formera un préalable indispensable à notre analyse critique.

Si l'on associait jusqu'ici l'œuvre à une méthode, celle de «faire des histoires^, ce qui distingue réellement Sophie Calle des autres artistes de sa génération est le genre performatif qu'elle explore en vue d'inventer un art de vivre, au sens où vivre serait une action ininterrompue, et non un mode. Emblématique de toute sa démarche, le souvenir de son action légendaire consistant à filer des inconnus 5 donne aujourd'hui du relief à cette quête assidue chez l'artiste des figures instables de la trace - où nous devinons une conception ontologique du médium photographique. Au début des années 1980, Jean Baudrillard lui lançait dans une postface: «Le réseau de l'autre est utilisé comme façon de vous absenter de vous-même. Vous n'existez que dans la trace de l'autre 6 .» À partir de cette invite, nous forgerons une approche de ce qui se manifeste dans l'image à travers les contours variables d'une place vacante, destinée non seulement à la projection identitaire du spectateur, mais aussi à l'observation de ce qui figure ni dans le texte ni hors de lui.

D’ordinaire, Sophie Calle attire l'attention sur ses procédés plutôt que sur l'envergure réflexive et théorique de son œuvre; elle désigne les moyens (la pratique introspective et autofictionnelle) pour mieux dissimuler les fins poursuivies en filigrane. En donnant à voir la complexité de l'interaction humaine, l'artiste

3. Parmi les titres imaginés pour l'exposition, Sophie Calle avait soumis celui de «Histoires à lire debout».

4. C'est Hervé Guibert qui, le premier, qualifia Sophie Calle de «faiseuse d'histoires» ("Panégyrique d'une faiseuse d'histoires», dans Sophie Calle. À suivre..., catalogue de l'exposition, Paris, ARC-Musée d'Art Moderne de la ville de Paris, 1991, n.p.). En 1991, Johanne Lamoureux et Annette Hurtig organisèrent à Toronto l'exposition «Faire des histoires », consacrée à Annette Messager, l'incontestable aînée de Sophie Calle en la matière. Voir : Annette Hurtig, Johanne Lamoureux (dirs.), Annette Messager, Faire des histoires, Toronto, Mercer Union Gallery, Cold City Gallery, 1991. Cette exposition fut ensuite présentée à la Mendel Art Gallery de Saskatoon, à la House Gallery et à la Contemporary Art Gallery, toutes deux de Vancouver.

5. Voir Cécile Camart, «De dérives en filatures: un érotisme de la séparation ", Esse, «Dérives II", n 55, automne 2005, p. 32-35, et «Sophie Calle, 1978-1981. Genèse d'une figure d'artiste», Cahiers du Musée national d'art moderne, n 85, automne 2003, p. $50-77$.

6. Jean Baudrillard, «Please Follow Me» (postface), dans Sophie Calle, Suite vénitienne, Paris, Éditions de l'Étoile, coll. «Écrits sur l'image », 1983, p. 81-93. 
tisse ses histoires autour des arcanes du lien, quelle qu'en soit la nature, éphémère, passionnelle, fraternelle ou communautaire. C'est à ce niveau qu'intervient l'ambivalence d'une absence-présence, notamment dans les situations construites en 2003. Chacune est traversée par la douleur, la séparation, le départ, le manque, l'échec, la disparition - leitmotive qui convergent vers la question obsédante de l'impossible adieu et du deuil impensable, et s'enracinent dans le secret de la trace, le motif de la relique, dans un sens nouveau cette fois. La structure mémorielle appréciable dans certaines pièces conçues par l'artiste dans les années 1990 concernait surtout une réflexion muséologique ou l'engagement politique et social des mémoires individuelle et collective7 ${ }^{7}$ C'est désormais l'expérience esthétique et conceptuelle du «manque» - s'exprimant en creux - qui agit comme déclencheur dans le processus créatif.

Rompue à l'usage d'une pratique photographique propre au champ de l'art contemporain ${ }^{8}$, Sophie Calle n'avait pourtant jamais véritablement sondé l'image en tant que médium autonome dans ses installations, tant sa présence semblait évidente. Produites directement, déléguées à un intermédiaire ou trouvées, les photographies venaient comme par magie s'agréger aux textes. La critique considérait étrangement comme secondaire cette composante essentielle des installations. Dans ses œuvres de 2003, Sophie Calle lutte puis abdique, sans mots, devant les images qu'elle réunit. Elle interroge leurs fonctions médiatiques, envisage leur échec. Lorsqu'elle remet en question la combinaison de ces médias c'est pour mieux céder au pouvoir évocateur et sidérant de la photographie et conclure à son autonomie. Le mutisme de l'image, sa disparition ou son processus de destruction affleurent. Alors que la narration est sciemment réduite à l'échelle anecdotique, signaler ce qui fait défaut - une idée (Unfinished), la clef d'une énigme (Une jeune femme disparaît), un amant (Douleur exquise; Voyage en Californie) - revient à énoncer une tension, un équilibre précaire entre le manque (donc le désir) et la perte (le deuil).

7. L'artiste a abordé cette matière dès 1994 avec l'exposition Absence (Rotterdam, Museum Boymans-van Beuningen, 1994) puis dans l'édition d'un coffret (L'absence, Arles, Actes Sud, 2000). Dans ce dernier sont réunies des pièces anciennes, Fantômes (1989), Last Seen [rebaptisée Disparition] (1991) et Souvenirs de Berlin-Est (1996), où règne une mise en abîme des espaces vacants (tableaux prêtés ou volés, insignes de l'ancienne RDA) au moyen de récits d’anonymes remaniés. Voir aussi Cathrin Pichler, «Der Spur auf der Spur, zur Erinnerungsarbeit in Projekten von Sophie Calle», Kunstforum International, octobre-décembre 1994, n 128, p. 227-233.

8. Dominique Baqué, La photographie plasticienne, Paris, Éditions du Regard, 1999. 


\section{UNFINISHED ET L'IMAGE EN MANQUE}

Sophie Calle raconte s'être procurée, en 1988, des bandes vidéos conservées dans un commissariat de police à Minneapolis. Prises par une caméra de surveillance cachée au-dessus d'un distributeur de billets, les séquences sont constituées de clichés saisis toutes les vingt secondes, enregistrant chaque opération bancaire en noir et blanc. Dans la succession des visages anonymes, l'artiste repère une palette d'émotions, de l'espoir à la joie, en passant par la crainte, la déception, la violence (fig. 1). Un rapprochement avec l'effet du miroir sans tain ne lui aura pas échappé. L'image inversée et le fantasme d'une vision de l'intérieur lui permettent de réactiver un des procédés dont elle a tiré, jadis, l'étrange plaisir de voir sans être $v u^{9}$. Quinze années lui auraient été nécessaires avant de trouver une juste destination à ces images. Elle déclare en effet s'être heurtée à une série d'échecs et d'insatisfactions. L'oscillation entre la quête de son style et la poursuite de l'achèvement de l'œuvre aurait présidé à la décision, en 2002, de tourner une vidéo qui relaterait les étapes de son investigation. La supposée défaillance de l'artiste se présente en ces termes: subit-elle une panne d'inspiration, ou pire, de génie? Pour la première fois, des images manquent de texte.

Je persistais à penser que ces images ne se suffisaient pas à elles-mêmes. Le texte manquait. Ce texte qui me colle à la peau. Ma marque de fabrique: image et texte. En montrant des documents trouvés, sans apport vécu de ma part, je ne collais pas à mon propre style ${ }^{10}$.

Il s'agit davantage de la crainte de manquer à la représentation qu'elle se fait de son langage artistique propre, et donc des modèles auxquels elle emprunte et qui constituent son outillage, comme le couple texte-image. En postulant la valeur intrinsèque de telles «images sans histoires », Sophie Calle reconsidère ses modes d'expression et par ricochet l'ensemble de son travail. Le titre même de la

9. Durant les années 1980, Sophie Calle se fait connaître avec des œuvres qui questionnent les thèmes de la surveillance et du pouvoir du regard. Aujourd'hui célèbres, ses filatures à Venise (Suite vénitienne, 1980) et à Paris (La filature, 1981) trouvent leur issue en 1983 avec L'hôtel, une situation plus radicale durant laquelle l'artiste espionne les occupants d'un hôtel après s'y être fait embaucher comme femme de chambre. En 1986, elle propose une pièce plus réflexive (Les aveugles) à partir de la rencontre avec des aveugles de naissance qu'elle interroge au sujet d'une impossible définition de la «beauté».

10. Sophie Calle, En finir, avec la collaboration de Fabio Balducci pour les dernières années, adapté du film Unfinished, réalisé par Sophie Calle et Fabio Balducci, Arles, Actes Sud, 2005, p. 55. La transcription éditoriale du scénario du film Unfinished produit du texte là où l'artiste disait en manquer. 


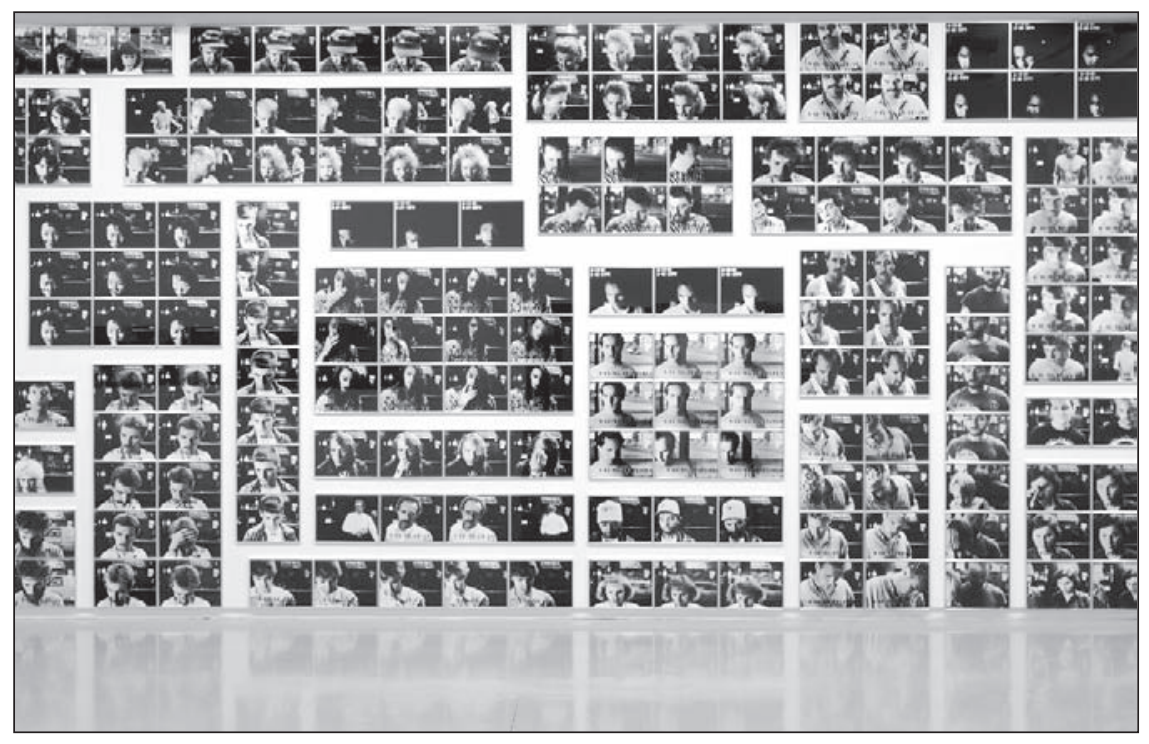

Fig. 1. Sophie Calle, Unfinished / Cash Machine, vue d'exposition, 2003, Galerie Emmanuel Perrotin, Paris. (C) Sophie Calle/SODRAC 2006. Gracieuseté de la Galerie Emmanuel Perrotin, Paris / Miami.

pièce porte cet idéal de finitude et d'achèvement et souligne le processus temporel de la création. Dans Le chef-d'œuvre inconnu, Balzac avait sondé les thèmes centraux de la genèse de l'œuvre, de la recherche du moment de vérité, des limites de l'artiste devant ses ambitions. Le vieux Frenhofer ne s'écriait-il pas: «Il ne suffit pas pour être un grand poète de savoir à fond la syntaxe et de ne pas faire de faute de langue ${ }^{11} »$ ? Sophie Calle concède «que ce n'était pas un problème de texte, de format ${ }^{12}$.» En lieu et place de commenter l'effet médusant de ces images devant lesquelles abdique le texte, ou de montrer ce qui fait fonctionner la pièce une fois aboutie, l'artiste renverse la difficulté et propose habilement non seulement de disséquer les défaillances du modèle, mais encore d'en faire le sujet annoncé de l'œuvre: «Tout est là : il s'agit de l'anatomie d’un échec. Ce fiasco accepté fait désormais partie du programme ${ }^{13}$.»

11. Honoré de Balzac, Le chef-d'œuvre inconnu [1830], suivi de Pierre Grassou, Sarrasine, Gambara et Massimila Doni, édition préfacée par Robert André, Paris, Éditions Gallimard et Librairie Générale Française, coll. «Le Livre de poche», 1970, p. 27.

12. Sophie Calle, En finir, p. 102.

13. Sophie Calle, En finir, p. 105. 


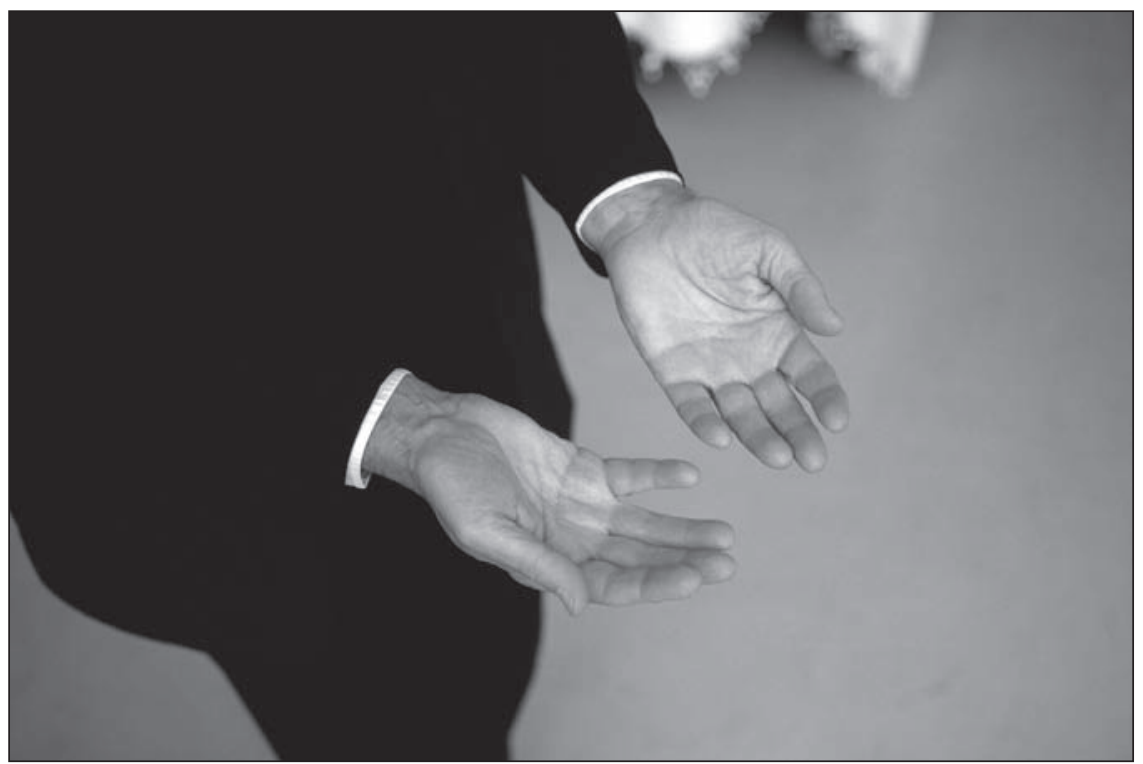

Fig. 2. Sophie Calle, Unfinished / Les mains qui ont touché l'argent, détail, 2003, Galerie Emmanuel Perrotin, Paris. (C) Sophie Calle/SODRAC 2006. Gracieuseté de la Galerie Emmanuel Perrotin, Paris/Miami.

Clef de voûte de l'exposition M'as-tu vue, le projet Unfinished comprend un film et trois séries d'images sans texte. La vaste cimaise nommée Cash machine se trouve entièrement saturée par plus de trois cents photographies noir et blanc et autant de visages muets associés en courtes séquences suivant un modèle pléthorique, qui déclenchent chez le spectateur un effet visuel de fascination et le laissent sans voix à son tour. Seule la mise en scène au musée permet d'en rendre compte - pour peu qu'on la compare avec l'édition du livre, privée d'une telle portée en raison de sa forme fixe - d'autant plus que le dispositif d'accrochage in situ connaît des aménagements possibles. En effet, les images sont disposées de manière à couvrir intégralement les parois de la pièce, en sorte que le spectateur éprouve une sensation d'immersion. Cette saturation délibérée traduit la volonté chez l'artiste de mener une réflexion sur les modes opératoires de la pratique photographique et en particulier l'usage de la série, telle que l'ont pratiquée les Becher. La répétition de ces visages suscite un rapprochement formel avec les portraits d'un Thomas Ruff, à la suite de l'école de Düsseldorf. Loin de ces choix, Sophie Calle introduit pourtant un rapport de force entre l'univers prosaïque et pathétiquement banal de ces images trafiquées - dont l'accumulation fait oublier la faible qualité - et la tentation d'esthétisation à laquelle elle ne peut échapper lorsqu'elle fixe des expressions universelles qui provoquent la projection et l'identification. 


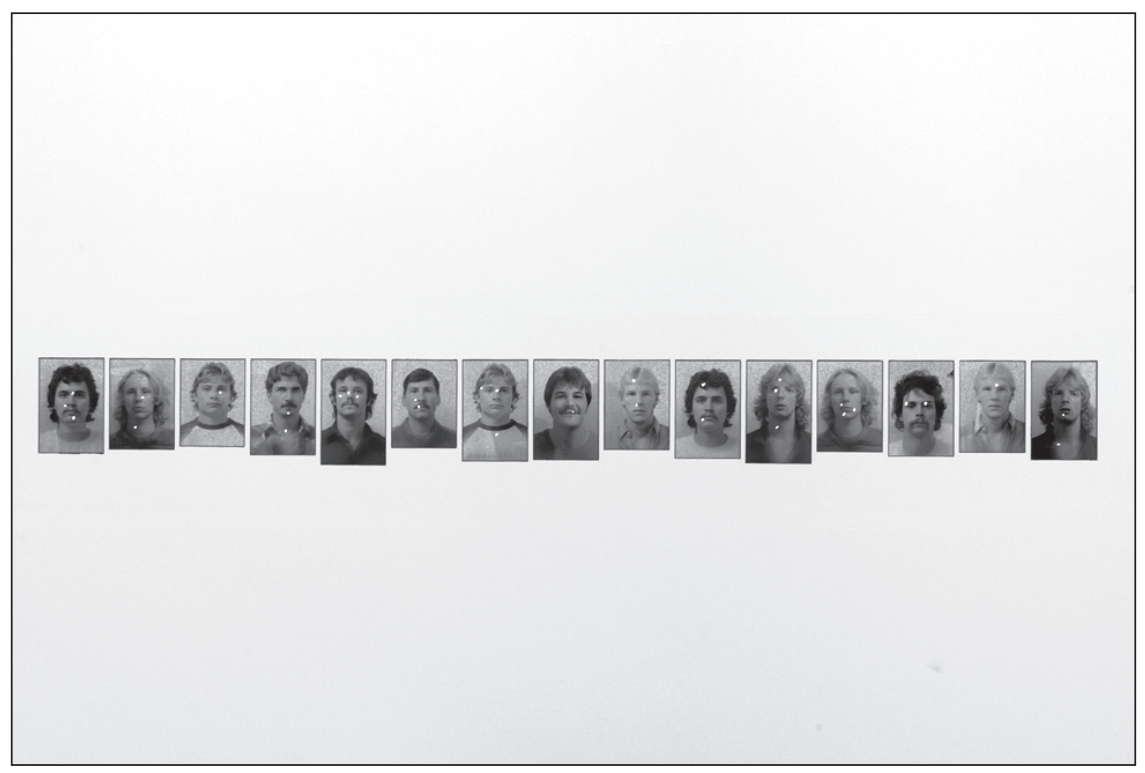

Fig. 3. Sophie Calle, Unfinished / Cours de cible, vue d'exposition, 2003, Galerie Emmanuel Perrotin, Paris. (C) Sophie Calle/SODRAC 2006. Gracieuseté de la Galerie Emmanuel Perrotin, Paris/Miami.

Des interviews menées par l'artiste auprès d'employés de banque ne seront qu'un point de départ pour une autre série d'images dans Unfinished, sous-titrée Les mains qui ont touché l'argent (fig. 2). Face à ces photos couleur de grand format, notre regard est happé par les paumes qui se tendent vers le vide sans autre commentaire, si ce n'est celui apporté par le film. Dans un article intitulé «Quand les mots font défaut», Rosalind Krauss commentait l'usage du motif de la main en photographie, envisagée dans les années 1920 et 1930 comme le symbole de l'espace corporel du photographe et du prolongement de son regard. La réflexion de l'auteur nous invite à penser que cette figure situe toujours l'image dans une relation problématique à l'écriture, car

la paume de la main, en tant qu'expression de l'élan spontané à réaliser et à laisser une trace, a obsédé la photographie et a usurpé le champ de l'image photographique, y gravant d'autre part la permanence de la trace écrite opposée à l'aspect fugitif de la simple image visuelle ${ }^{14}$.

Suivant une approche beaucoup moins surréaliste malgré son titre Cœurs de cible, le dernier opus de l'ensemble présente quant à lui une succession de

14. Rosalind Krauss, "Quand les mots font défaut», trad. Marc Bloch, Critique, n 459-46o, août-septembre 1985, p. 759. 
portraits en couleur de délinquants ayant servi de cibles pour des séances de tir dans un commissariat de police américain. Les visages sont alignés, incrustés dans la cimaise et combinés à une source de lumière blanche et vive qui surgit depuis l'arrière-plan, traverse et met en évidence les trous dans les photographies (fig. 3). Plus près, nous observons que la surface traversée par les impacts dévoile les couches intermédiaires du papier. Des fragments de l'image emportés par la trajectoire de la balle sont ainsi soustraits à la vue, ils ont disparu, des morceaux de visages manquent à ces portraits. L'anonymat de ces adolescents et le contexte morbide du dispositif trouvent une résonance dans certains projets menés par Christian Boltanski sur le thème de la mémoire. Laspect sensationnel était totalement exploité dans ses Archives de l'année 1987 du journal El Caso (1989). Pour cette pièce, il avait rephotographié en gros plan des portraits de famille de personnes assassinées, publiés par la presse à scandale espagnole. Mais nous pensons encore aux Suisses morts, série de travaux entamée en 1990, où interviennent des portraits issus de la rubrique nécrologique d'un journal suisse. L'emploi récurrent des images trouvées et la violence macabre qu'elles contiennent forment les points de contact entre les univers respectifs des deux artistes.

Une variation substantielle a caractérisé les Cœurs de cible présentés chez Emmanuel Perrotin à l'automne 2003. Tandis que le Centre Pompidou montrait les tirages originaux, selon les dires de Sophie Calle, la galerie proposait des fac-similés perforés artificiellement. De surcroît, une bande de plastique blanc masquait les yeux des délinquants, précaution prise prétendument pour éviter les aléas judiciaires relatifs au droit à l'image. S'agit-il d'un jeu supplémentaire avec les mots? Pourquoi l'artiste introduit-elle soudain cette distinction - surannée, elle le sait - entre la matrice et la copie? L'interdiction mystérieuse de voir le regard ajoute une grille d'interprétation devant celle des perforations de l'image atteinte dans sa matérialité même. De cette comparaison entre les deux versions d'une seule œuvre surgit une mise en abîme de la photographie, qui dupe celui qui la contemple.

L'artiste montra enfin la même année une série plus confidentielle ${ }^{15}$. Variation sur le même thème, Cash Machine, l'agression de Pamela Magnuson

15. Lors de l'exposition collective Par le chas de l'aiguille, à la galerie Chantal Crousel (Paris, été 2003). Cette série n’est pas intégrée à l'ensemble Unfinished. Chantal Crousel l'avait déjà montrée au printemps 1991 dans l'exposition Vanitas. Elle fut ensuite exposée au Musée de la Poste lors de la manifestation Les couleurs de l'argent durant l'hiver 1991-1992, puis au printemps 1992 à la Hayward Gallery de Londres, dans le cadre de l'exposition Doubletake: Collective Memory and Current Art. 
le 8 août 1983 à 21 heures 54 minutes et 20 secondes désigne quatre clichés où l'on voit disparaître une femme assommée par un agresseur auquel succède un homme, torse nu. Trois visages seulement, à l'expression indéfinissable, entre lesquels s'intercale une image sombre, un peu floue, quasiment abstraite. Quelque chose manque: la femme est littéralement éclipsée, si bien que l'agression prend la forme d'un défaut de représentation, d'une rupture dans la séquence. Comme devant les images d'Une jeune femme disparaît, le spectateur doit imaginer la scène à travers les formes, mais aussi dans les intervalles temporels horodatés. Cette substitution d'une personne par une autre nous apparaît comme une nette allusion à l'identité fragmentaire de l'artiste qui distribue ses facettes entre les protagonistes de ses œuvres. D’un point de vue formel, l'image constitue ici la preuve du méfait, l'unique trace de ce qui est réellement arrivé à la cliente agressée. Le statut testimonial du médium photographique est confirmé, mais nous observons un déplacement du champ de la performance stricto sensu vers le registre du fait divers et du sensationnel : l'action n'est pas générée ou vécue par l'artiste mais surveillée.

\section{BÉNÉDICTE OU LA DISPARITION DANS L'IMAGE}

À l'automne 2003, les visiteurs du Centre Pompidou croisaient des avis de recherche que l'artiste avait fait épingler dans l'ensemble des espaces dévolus au public. Hommage rendu à une jeune femme disparue, autrefois gardienne d'exposition au Musée national d'art moderne? Conjuration du sort pour tenter de la faire revenir? Un goût pour l'enquête a sans doute attiré Sophie Calle sur le terrain délicat des affaires de disparitions non résolues. Cette histoire trouve son origine en 2001, dans une œuvre intitulée Vingt ans après, remake assumé de La filature (1981) formant un tissu d'intertextualité. Sophie Calle y relate un fait divers, annonçant son dessein artistique:

Dans la nuit du 26 février 2000, peu après l'incendie de son habitation, une jeune fille du quartier a disparu. Elle a dévalé, pieds nus, les escaliers, sous le regard des pompiers. On ne l'a jamais revue. Bénédicte V. aurait écrit dans son journal intime qu'elle voulait vivre comme moi. Un inspecteur m’a contactée. [...] Mêlée à ce fait divers, j’ai à mon tour rencontré ses amis, visité sa maison ravagée. J'en ai rapporté des cendres, des négatifs calcinés, et des photographies ${ }^{16}$.

16. Extrait du texte de Vingt ans après (2001), non publié, avec l'aimable autorisation de Sophie Calle et de la Galerie Emmanuel Perrotin, Paris/Miami. 

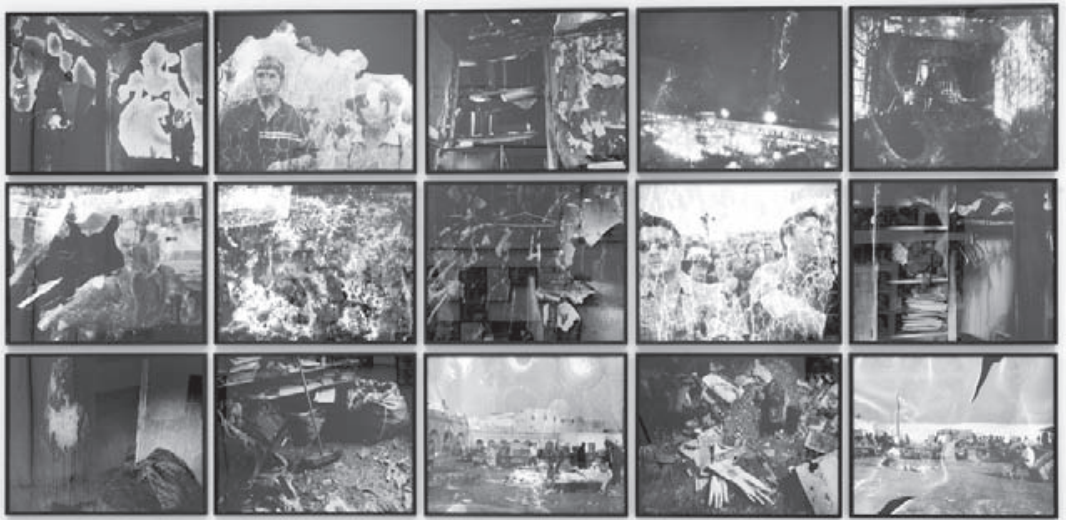

Fig. 4. Sophie Calle, Une jeune femme disparaît, vue d'exposition, 2003, Galerie Emmanuel Perrotin, Paris. (C) Sophie Calle/SODRAC 2006. Gracieuseté de la Galerie Emmanuel Perrotin, Paris/Miami.

Traduction muséale de cette annonce, Une jeune femme disparaît (2003) est une théâtralisation du vide et de l'absence (figs. 4-5). Dans un coin de la pièce, une chaise de gardien inoccupée dessine la présence in absentia de la disparue. Cette mise en scène peut paraître prosaïque mais elle fonctionne selon nous comme une métonymie du manque en tant que matière, comme un déplacement de l'image, ainsi que le suggère Jean-Luc Nancy:

La place vide de l'absent comme place non vide, voilà l'image. Place non vide ne veut pas dire place remplie: cela veut dire place de l'image, c'est-à-dire en fin de compte l'image en tant que place, et place singulière de ce qui n'a pas de place ici: place d'un déplacement, métaphore, nous y revoilà ${ }^{17}$.

Sur l'un des murs, les fragments choisis d'un article de presse flottent dans un espace blanc encadré. Puis il faut nous approcher d'une petite vitrine en forme d'écrin pour découvrir une boîte de diapositives calcinées, au plastique fondu et recroquevillé. Ce dispositif présente tous les aspects de la relique, dans le sens délivré par Pierre Fédida: «ce qui, du mort, est conservé pour garantir,

17. Jean-Luc Nancy, «L'oscillation distincte», dans Sans commune mesure. Images et textes dans l'art actuel, catalogue d'exposition, Paris, Éditions Léo Scheer, 2002, p. 10. 


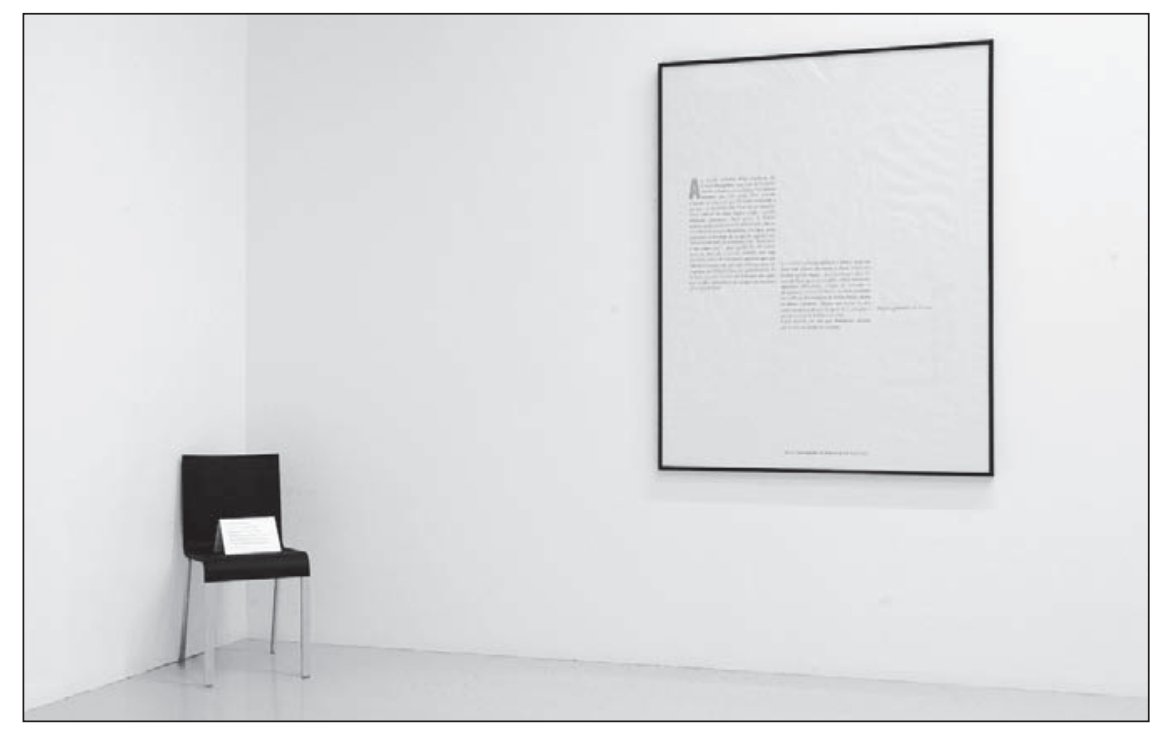

Fig. 5. Sophie Calle, Une jeune femme disparaît, vue d'exposition, 2003, Galerie Emmanuel Perrotin, Paris. (C) Sophie Calle/SODRAC 2006. Gracieuseté de la Galerie Emmanuel Perrotin, Paris/Miami.

au nom de la réalité, qu'il ne reviendra pas $^{18}$. » Ici, la relation à l'objet se définit à travers la séparation et l'acceptation de la perte.

Parce qu'elle recueille, dans la matérialité d’un reste familier autant que dérisoire, l'étrange vertu du corps absent, la relique donne son droit de nécessité et par le rituel du culte privé qu'elle instaure, défie, dans le travail de deuil, les apparences de la mort. [...] la relique prend sens dans le désir de conserver quelque chose de ce dont on se sépare sans, pour autant, devoir renoncer à s'en séparer ${ }^{19}$.

La plus grande cimaise est réservée à un vaste «damier», alternance d'images couleur et noir et blanc, quasi abstraites. Dans l'appartement sinistré, l'artiste a récupéré des films photographiques appartenant à la jeune femme. Elle ranime ces images éteintes, fait développer ces négatifs dégradés par la chaleur des flammes. Au moyen des films négatifs, c'est la matérialité du médium qui est concernée, au point d'y déceler une défense discrète de l'argentique à l'ère du numérique. La réflexion de l'artiste sur le statut de l'image photographique se prolonge encore, sous la forme d'une évocation discrète des différents registres

18. Pierre Fédida, Labsence, Paris, Éditions Gallimard, 1978, p. 53.

19. Pierre Fédida, Labsence, p. 53. 


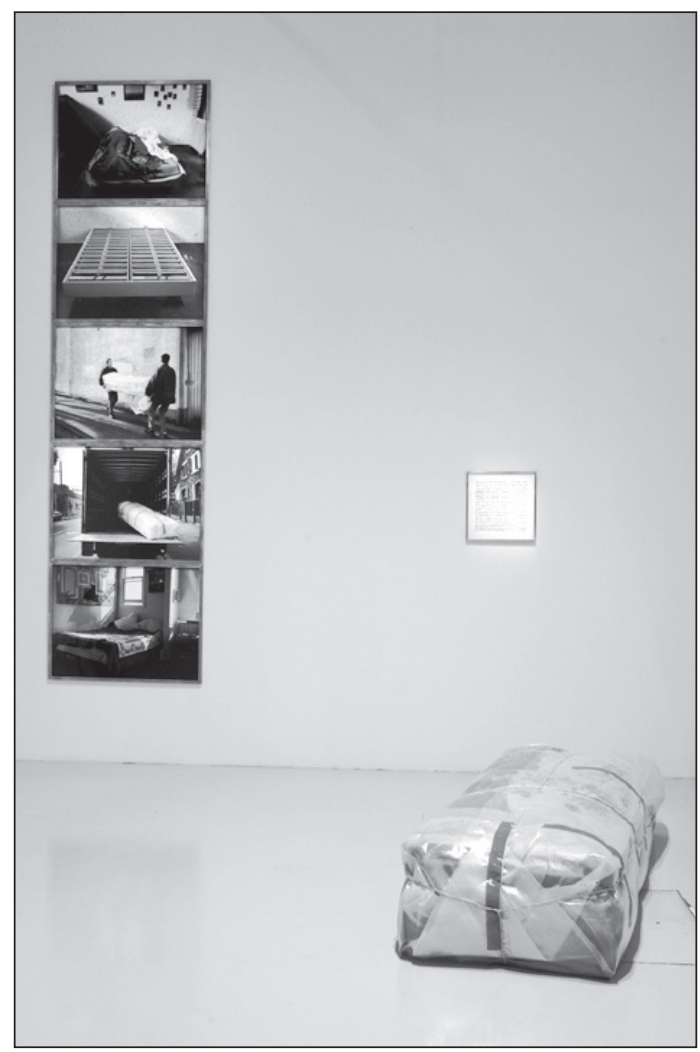

Fig. 6. Sophie Calle, Voyage en Californie, vue d'exposition, 2003, Galerie Emmanuel Perrotin, Paris. (C) Sophie Calle/ SODRAC 2006. Gracieuseté de la Galerie Emmanuel Perrotin, Paris/Miami.

envisageables - images trouvées, abîmées, modifiées, manipulées. Il en résulte ces images troublantes prises par la disparue lors de voyages à l'étranger, où l'on devine des silhouettes, des paysages, des vues d'architectures. Ces images en noir et blanc dévoilent les altérations subies par le celluloïd - ondulations de la surface, déchirures, fragments lacunaires - et les nombreuses zones floues, indéterminées. Dans un récent essai, François Noudelman soulignait l'ambivalence qui sous-tend la visibilité de l'image, laquelle «met en ouvre une absence active aussi bien du référent visible que du sujet voyant ${ }^{2 \circ} »$. Ainsi l'image serait une mise

20. François Noudelman, Image et absence. Essai sur le regard, Paris, Montréal, L'Harmattan, 1998, p. 9. 
L'ABDICATION DEVANT L'IMAGE?

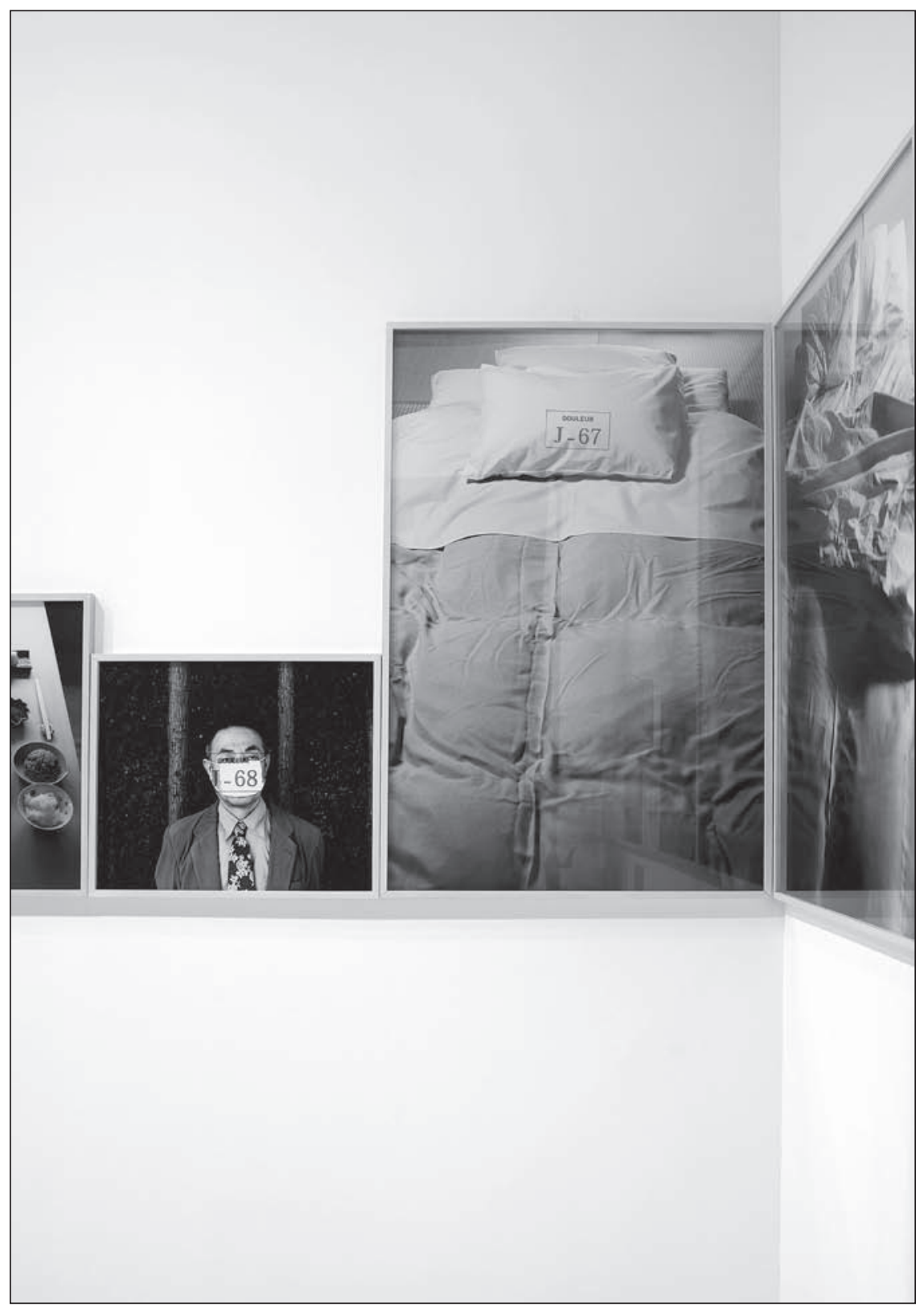

Fig. 7. Sophie Calle, Douleur exquise. Avant la douleur, détail, J-68 J-67, 1984-2003, Galerie Perrotin, Paris. (c) Sophie Calle/SODRAC (2006). Gracieuseté de la Galerie Emmanuel Perrotin, Paris/Miami. 


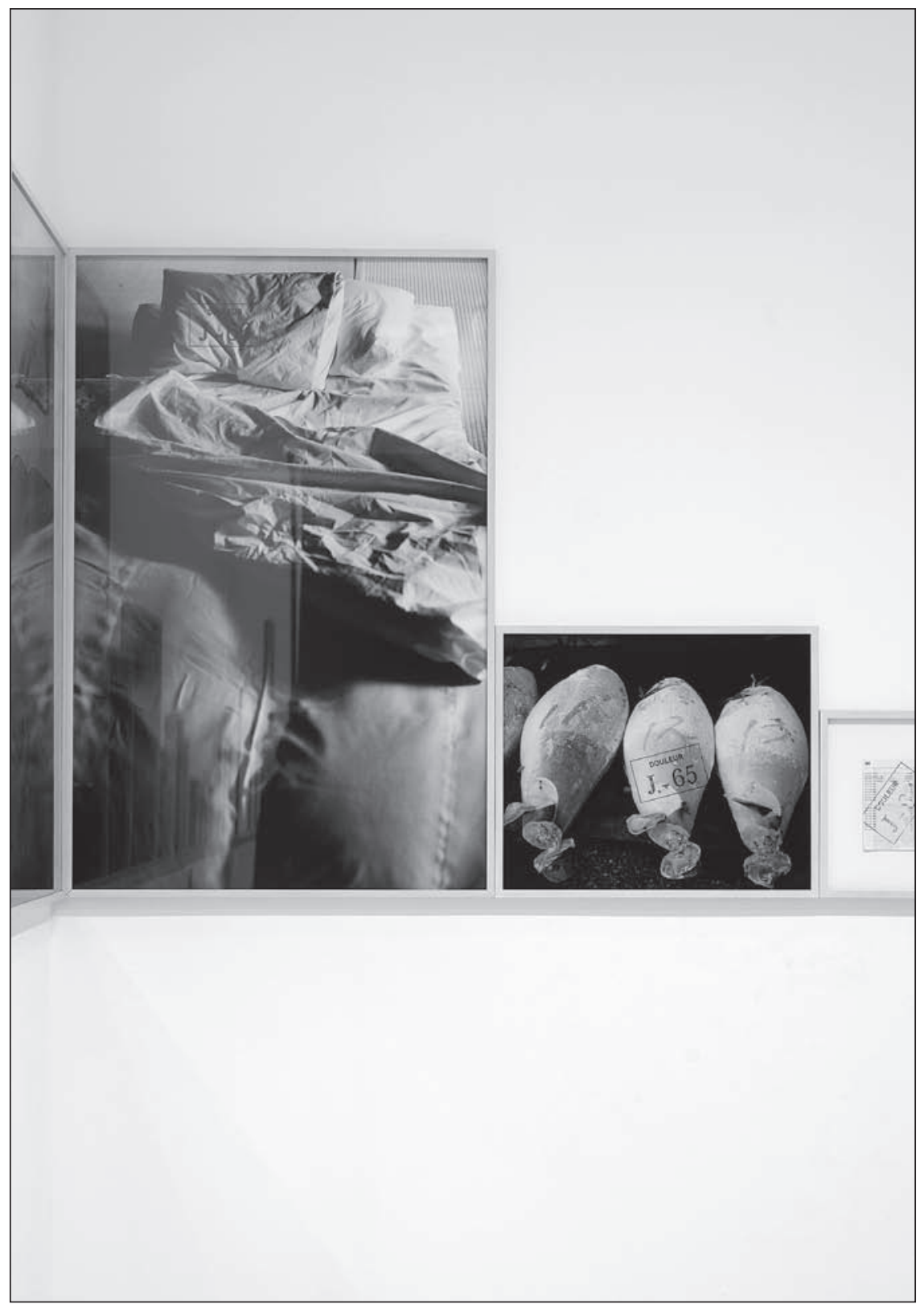

Fig. 8. Sophie Calle, Douleur exquise. Avant la douleur, détail, J-66 J-65, 1984-2003, Galerie Emmanuel Perrotin, Paris. @ Sophie Calle/SODRAC (2006). Gracieuseté de la Galerie Emmanuel Perrotin, Paris/ Miami. 
en scène de l'absent: comme représentation, elle est un substitut qui comble un déficit de réalité. Mais selon l'auteur, l'image se distingue de la relique; en effet:

[...] ce qui reste en elle de l'objet met en jeu un procès, une ouverture beaucoup moins tangible et moins visible, car elle suppose un échappement du représentant impossible à cerner, toujours échappant ${ }^{21}$.

À son tour, Sophie Calle a photographié l'appartement, en couleur. Les flammes ont léché les surfaces, formant des paysages sombres et abstraits qui exposent les murs à nu, sous les lambeaux de tapisserie, les couches de peinture écaillée. L'artiste semble hypnotisée par les reliques d'une vie suspendue, ici un cintre seul dans une penderie carbonisée, là quelques livres roussis sur une étagère, plus loin des objets méconnaissables jonchant le sol. Selon une asymétrie maîtrisée, la composition fait se croiser deux trames narratives, deux visions. La destruction de l'image saisit d'effroi le spectateur, contraint à plonger dans ces abîmes. Sophie Calle fabrique des images à partir d'oripeaux détériorés et manquants. Elle s'engage à une renaissance de ces images contre leur disparition qui ferait craindre, comme Daniel Sibony le souligne, que :

[l'événement] n'a pas eu lieu: rien n'est là pour le nommer, le pointer, lui donner corps. Il n'y a qu'un vide, même pas un négatif, même pas une ombre; comme si tout le vide qu'un corps peut occuper ou faire vivre s'étalait là, mais sans le corps, inerte. Naturellement, le corps n’est pas «mort» - même s'il l'est dans le réel; sa mort reste indicible: il n'y a aucune preuve de sa mort ni du fait qu'une personne l'a fait disparaître. Il est disparu. Il est retiré de l'être ${ }^{22}$.

\section{L'EMPREINTE EN CREUX DANS VOYAGE EN CALIFORNIE ET DOULEUR EXQUISE}

Le processus d'effacement de l'image par les flammes captive Sophie Calle depuis longtemps. Dans une de ses Histoires vraies intitulée Le lit (1988), elle prétend que sa mère avait loué sa chambre de jeune fille à un homme qui se donna la mort par immolation. Abandonné par les pompiers, le matelas brûlé est devenu une relique pour l'artiste et l'instrument d'un rite de passage. Tel un point d'ancrage de la mythologie callienne, ce premier deuil en augure d'autres.

Parfois, le registre est humoristique, comme en témoigne le texte de Voyage en Californie (2003), une drolatique démonstration d'empathie:

21. François Noudelman, Image et absence, p. 147.

22. Daniel Sibony, «La disparition », Événements II. Psychopathologie du quotidien, Paris, Éditions du Seuil, coll. «Points Essais», 1995, p. 31. 
J'ai reçu une lettre de Californie : «4 juin 1999. Chère Mme Calle, je suis un Américain de vingt-sept ans. J'ai vécu une longue idylle qui s'est récemment dénouée. J'aimerais passer le reliquat de cette période de deuil, d'affliction, dans votre lit...» Accepter se révélait délicat. Considérant la distance qu'il lui faudrait parcourir, si l'inconnu me déplaisait, pouvais-je décemment le congédier? Et puis il y avait déjà un homme dans mon lit. Deux mois plus tard, ma literie prit l'avion pour San Francisco. [...] Je souhaitais au destinataire un prompt rétablissement [...] En septembre, j’appris que la souffrance s'estompait. Le 2 février 2000, mon lit était de retour à la maison ${ }^{23}$.

L'installation présente un lit emballé, une série de photographies couleur illustrant le déménagement, puis quelques lettres et courriers électroniques attestant la correspondance échangée durant cette période (fig. 6). Dans la salle, le spectateur est contraint physiquement de faire le tour de ce lit empaqueté, massif, compact, qui matérialise à la fois le poids de l'absence et la présence fantasmatique de l'artiste. Décidément seul, le Californien ne retrouve ni l'amoureuse perdue, ni Sophie Calle. Sur un mode plus léger, l’artiste renoue ainsi avec une thématique abordée jadis dans Les dormeurs (1979). Elle envisage cette fois le sommeil comme une absence à soi-même, une échappée du monde réel où peut se consommer le deuil. La dimension performative de son art est également mise en évidence: l'œuvre est le résultat d'une action à laquelle le spectateur n'a pas assisté.

Un des récents mythes élaborés par l'artiste est l'expérience d'un deuil amoureux vécu à l'occasion d'un voyage dans les années 1980. L'intensité dramatique est ancrée dans le ton choisi dès l'introduction de Douleur exquise (1984-2003):

En 1984, le ministère des Affaires étrangères m’a accordé une bourse d'études de trois mois au Japon. Je suis partie le 25 octobre sans savoir que cette date marquait le début d'un compte à rebours de quatre-vingt-douze jours qui allait aboutir à une rupture, banale, mais que j’ai vécue alors comme le moment le plus douloureux de ma vie ${ }^{24}$.

Seule la première partie de l'installation attire ici notre attention. Laccrochage linéaire en forme de cheminement chronologique est réparti selon les cas dans plusieurs salles, suivant une construction narrative typiquement romanesque. Chaque jour égrené est symbolisé par une image - photographie, document de voyage ou lettre - qui conduit à rebours jusqu'à l'acmé de la frise: le

23. Sophie Calle, Voyage en Californie, avec la collaboration de Josh Greene, dans M'as-tu vue, catalogue de l'exposition, Christine Macel (éd.), Paris, Éditions du Centre Pompidou, Éditions Xavier Barral, 2003, p. 198.

24. Sophie Calle, Douleur exquise, Arles, Actes Sud, 2003, p. 13. 
jour de la rupture. Le jour J-63 avant la douleur raconte une dispute déclenchée par la perte d'un portrait de Sophie Calle enfant, que l'artiste aurait confié à l'écrivain Hervé Guibert. L'artiste se situe ici dans une perspective barthésienne d'un «ça-a-été » réconfortant de la photographie. Mais elle met en exergue les trous de la mémoire, associant la perte d'une image de soi au deuil de l'identité. Cette «photo manquante» qui échappe au spectateur éveille un parallèle avec L'amant de Marguerite Duras. Pour des motifs étranges de retirage difficile, l'image perdue (puis retrouvée) ne figure pas au musée, seulement dans l'édition du livre ${ }^{25}$. Ce type de microvariations est une méthode caractéristique de l'artiste qui remet l'image en question.

Les pérégrinations ne font qu'accentuer le sentiment d'une perte, d'une fuite en avant hors d'elle-même. L'image d'un boulier renforce cette angoisse croissante, à mesure que les retrouvailles présumées approchent. Le motif de l'empreinte en creux se manifeste durant toute cette attente. Dès sa première œuvre, Les dormeurs (1979), le motif du lit - lieu de l'intimité et de l'attente cristallise toutes les variations sur le discours amoureux. Dans No Sex Last Night (1992), elle filmait avec dépit l'empreinte des corps dans les draps défaits des chastes nuits passées avec son compagnon Greg Shepard. Pour le jour J-70, l'artiste photographie un oreiller, auquel succède trois jours plus tard une paire de lits jumeaux, dont un seul est défait, signe du passage de la dormeuse esseulée (figs. 7-8). L'autre lit semble aussi vide que celui paré de draps à fleurs bleues, sur l'image $J$-8.

Deux jours avant «la douleur», Sophie Calle figure sur une photographie, radieuse, assise dans un bar. Nous distinguons nettement le bras d'un homme enlaçant son épaule droite, mais l'image a été découpée, si bien que nous ne voyons pas cet homme. Tout laisse à penser que c'est le protagoniste du récit qui fait défaut. L'artiste transforme un cliché antérieur à la rupture et accélère l'action dramatique en suggérant la suite. L'image est utilisée comme un fragment du temps qui se découpe, se manipule et participe à une réécriture du passé. Elle devient le lieu de l'effacement d'un personnage, puisque celui-ci s'échappe du récit, puisqu'il échappe finalement à l'auteure. Sophie Calle use de cette parade - amputer l'image de la figure masculine, souligner la carence. Finalement, l'homme a laissé quelque chose, de l'autre côté du miroir. À lui aussi, il manque quelque chose, il manque un bras, une main. Dans l'instant suivant, le spectateur doit se frayer un passage dans le Lieu de la douleur, une vraie fausse chambre d'hôtel qui renvoie à l'expérience du vide.

25. Sophie Calle, Douleur exquise, p. 73. 
Dans l'œuvre comme dans la vie, le deuil produit des alvéoles. Ces cavités dans la structure déclenchent une dynamique de circulation - du désir et du regard. Car il est question ici de désir scopique. L'image vient en renfort derrière l'insoutenable perte, pour tenter de figurer l'invisible ou l'indicible, contre la perte de vue. Lorsque les mots sont en excès, l'image s'hypertrophie et prend son autonomie. Quelle que soit la nature de la disparition à l'image, du registre anecdotique au plus dramatique, ces œuvres nous plongent au cœur de l'incapacité à dire au revoir, de l'impossible deuil. Bien qu'elles ne constituent nullement des actes de commémoration, leur dimension existentielle pose la question d'un « devoir de mémoire», et ainsi que le suggère Emmanuel Kattan, il s’agirait alors de repenser un «devoir du souvenir des morts ${ }^{26}$ ». Hors des dépôts officiels de la mémoire, hors des lieux de culte religieux, les pièces de Sophie Calle ont choisi le musée pour reliquaire.

26. Emmanuel Kattan, Penser le devoir de mémoire, Paris, Presses universitaires de France, 2002, p. 13 . 